\title{
Studying hot QCD matter at the CERN-LHC with heavy quarks
}

\author{
André Mischke* \\ ERC-Research Group QGP-ALICE, \\ Utrecht University, Princetonplein 5, 3584 CS Utrecht, the Netherlands \\ E-mail: a.mischkeduu.nl
}

This paper discusses selected highlights on open heavy-flavour production in pp and lead-lead collisions at the Large Hadron Collider (LHC) at CERN. Besides testing fundamental predictions from perturbative Quantum Chromodynamics (pQCD), elementary proton-proton interactions serves as an important baseline for studies in heavy-ion collisions. QCD predicts that in such heavy-ion collisions the formation of a deconfined state of matter, the Quark Gluon Plasma, can be reached at high temperatures and high energy densities. Heavy quarks are powerful probes to investigate this state of matter, since they are predominantly produced in the initial hard scattering processes, interact with the coloured medium and carry this information to the final state.

XXI International Workshop on Deep-Inelastic Scattering and Related Subject -DIS2013, 22-26 April 2013

Marseilles, France

\footnotetext{
* Speaker.
} 


\section{Introduction}

Relativistic nucleus-nucleus collisions allow exploring the behaviour of strongly interacting matter at high temperatures and high energy densities, where a new phase of matter, the Quark-Gluon Plasma (QGP), is predicted by Quantum Chromodynamics (QCD) to exist. In this phase colour confinement of quarks and gluons into hadrons should vanish. An overview of the latest heavy ion results at the LHC is given in [1,2]. Heavy quarks (charm and beauty) are sensitive probes to study the properties of the QGP. Due to their large mass $\left(m_{\mathrm{c}} \approx 1.5 \mathrm{GeV} / \mathrm{c}^{2}\right)$, heavy quarks are produced predominantly in the (hottest) initial phase of the collision via gluon fusion processes [3] and therefore allow to explore the complete space-time evolution of the QGP matter. This production process also dominates in heavy ion collisions where many (in part overlapping) nucleon-nucleon collisions occur. Thermal processes later in the collision might contribute to heavy-quark production at low transverse momentum [4]. These heavy quarks propagate through the hot and dense QCD matter and lose energy through medium-induced gluon radiation (colour charge dependent) and collisions with the medium. Moreover, theoretical models predicted that heavy quarks should experience smaller energy loss than light quarks due to the suppression of small angle gluon radiation (dead-cone effect [5, 6]). Thus, the study of heavy-flavour production in nucleus-nucleus collisions provides key tests of parton energy-loss models, yielding profound insight into the properties of the produced QCD matter.

\section{The baseline: Open heavy flavour production in elementary pp interactions}

A description of the Large Hadron Collider and the setup and performance of the ALICE, ATLAS and CMS experiments can be found in [7]. Heavy-flavour particles are measured via full reconstruction of $\mathrm{D}$ meson hadronic decay channels $[8,9]$ and via semi-leptonic decay channels [10]. The D meson yields were measured with an invariant mass analysis of reconstructed decay topologies, selected by means of topological cuts and particle identification (PID).
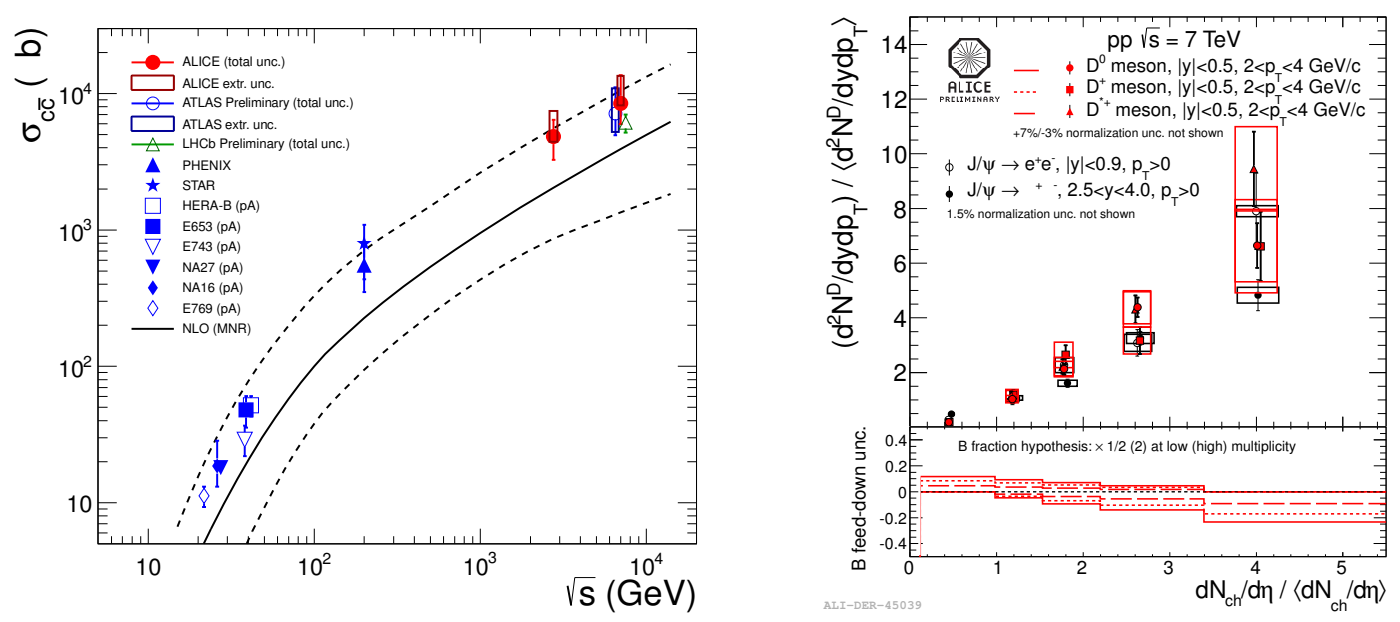

Figure 1: Left: Total nucleon-nucleon charm production cross section versus collision energy [8, 9]. The NLO MNR calculation [11] (and its uncertainties) is shown by solid (dashed) lines. Right: Relative yield for $\mathrm{D}^{0}, \mathrm{D}^{+}$and $\mathrm{D}^{*+}$ for $2<p_{\mathrm{T}}<4 \mathrm{GeV} / c$ and inclusive $J / \psi$ versus charged particles multiplicity. 

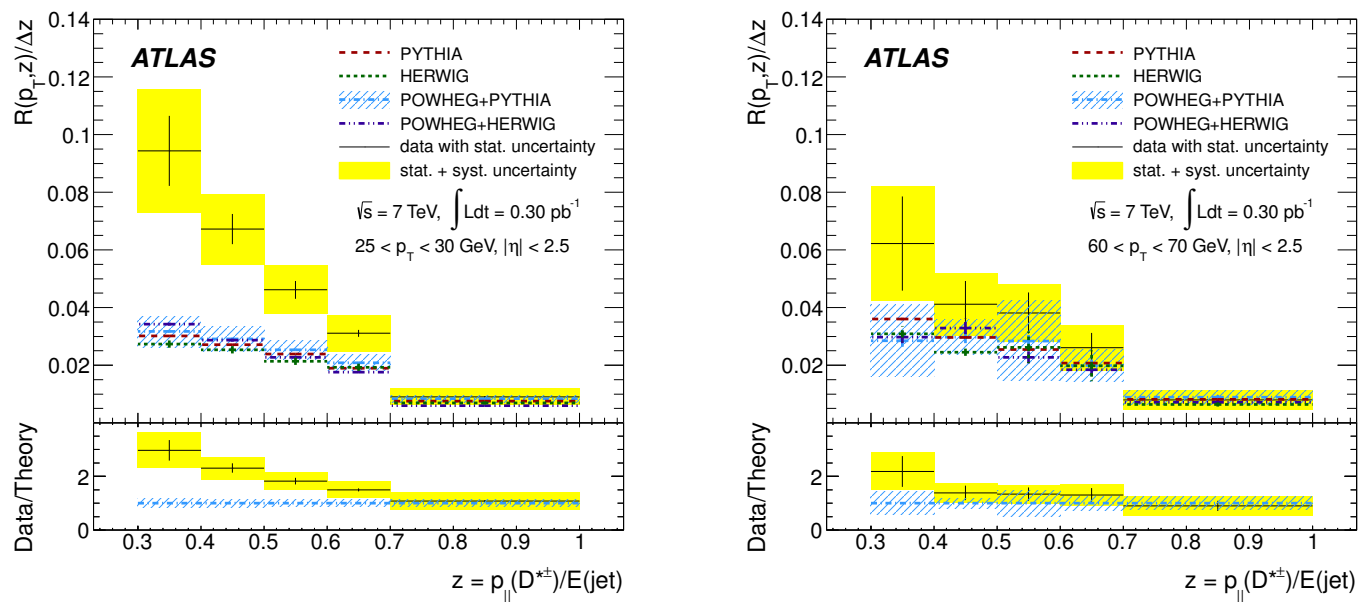

Figure 2: $\mathrm{D}$ production rate $R\left(p_{\mathrm{T}}, z\right) / \Delta z$ in the jet $p_{\mathrm{T}}$ range $25-30 \mathrm{GeV}$ (left panel) and $60-70 \mathrm{GeV}$ (right panel) versus $z$, measured in $7 \mathrm{TeV}$ pp interactions by ATLAS [13]. The data are compared with the predictions of the Monte Carlo event generators PYTHIA, HERWIG, POWHEG+PYTHIA and POWHEG+HERWIG. The insets show the ratio of the measurement to the POWHEG+PYTHIA prediction.

Figure 1, left panel, shows the collision energy dependence of the total nucleon-nucleon charm production cross section. The data are well described by the upper limit of the next-to-leading order MNR calculation [11]. Moreover, as illustrated in Fig. 1, right panel, the relative yield for D mesons in the $p_{\mathrm{T}}$ range $2-4 \mathrm{GeV} / c$ increase linearly with the charged particle density. Inclusive $J / \psi$ follows this trend. The data might be interpreted in terms of multi-parton interaction in the initial stage of the collision [12]. In Fig. 3 the production rate of $\mathrm{D}^{*+}$ mesons in jets in $7 \mathrm{TeV}$ pp collisions is depicted for two different jet energies [13]. Large discrepancies are observed between data and Monte Carlo predictions for low $z$, decreasing a little at higher $p_{\mathrm{T}}$. The measured $z$ distributions differ from the predictions of all considered generators considered both in overall normalization and shape. These observations indicate that the production of $\mathrm{c}$ jets (b jets) or their fragmentation into D mesons is not well modeled in current MC generators. Further QCD refinements are needed to improve the description of high- $p_{\mathrm{T}} \mathrm{D}$ meson production in the TeV energy range.

\section{Open heavy flavour production in lead-lead collisions at $\sqrt{s_{\mathrm{NN}}}=2.76 \mathrm{TeV}$}

Medium effects are typically quantified using the nuclear modification factor $R_{\mathrm{AA}}$ where the particle yield in $\mathrm{Pb}-\mathrm{Pb}$ collisions is divided by the yield in $\mathrm{pp}$ reactions scaled by the number of binary collisions. $R_{\mathrm{AA}}=1$ would indicate that no nuclear effects, such as Cronin effect, shadowing or gluon saturation, are present and that nucleus-nucleus collisions can be considered as an incoherent superposition of nucleon-nucleon interactions. By comparing the nuclear modification factor of charged pions $\left(R_{\mathrm{AA}}^{\pi^{ \pm}}\right)$, mostly originating from gluon fragmentation at this collision energy, with that of hadrons with charm $R_{\mathrm{AA}}^{\mathrm{D}}$ and beauty $R_{\mathrm{AA}}^{\mathrm{B}}$ the dependence of the energy loss on the parton nature (quark/gluon) and mass can be investigated [14]. A mass ordering pattern $R_{\mathrm{AA}}^{\pi^{ \pm}}<R_{\mathrm{AA}}^{\mathrm{D}}<R_{\mathrm{AA}}^{\mathrm{B}}$ is expected. Figure 3, left panel, depicts the $R_{\mathrm{AA}}$ for prompt $\mathrm{D}^{0}, \mathrm{D}^{+}, \mathrm{D}^{*+}$ and $\mathrm{D}_{\mathrm{S}}^{+}$mesons at mid-rapidity in central lead-lead collisions at $\sqrt{s_{\mathrm{NN}}}=2.76 \mathrm{TeV}$. All D mesons show a strong suppression of their yield (factor of $\approx 5$ at around $10 \mathrm{GeV} / \mathrm{c}$ ) and follow a similar trend at 

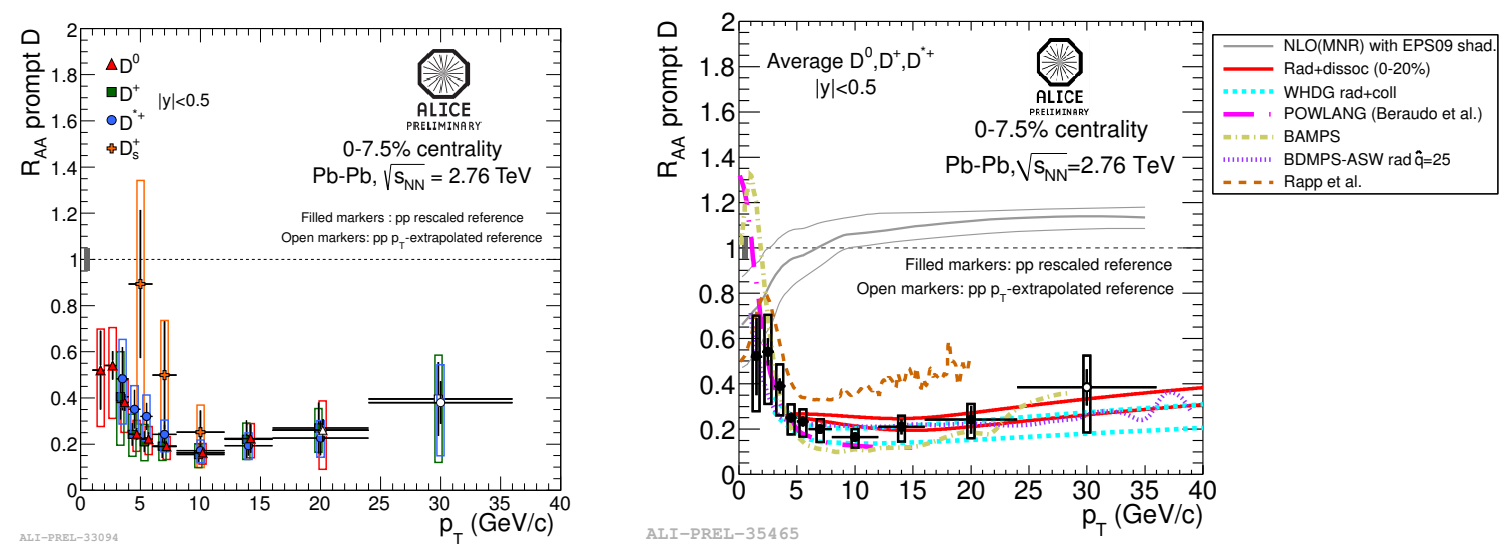

Figure 3: Left: $R_{\mathrm{AA}}$ of prompt $\mathrm{D}^{0}, \mathrm{D}^{+}, \mathrm{D}^{*+}$ and $\mathrm{D}_{\mathrm{s}}^{+}$mesons at mid-rapidity in the $0-7.5 \%$ centrality class of lead-lead collisions at $\sqrt{s_{\mathrm{NN}}}=2.76 \mathrm{TeV}$. Right: Average $R_{\mathrm{AA}}$ of prompt D mesons, compared to next-toleading-order pQCD calculations with nuclear shadowing [16] and different parton energy-loss models [15].

low $p_{\mathrm{T}}$, except the $\mathrm{D}_{\mathrm{s}}^{+}$mesons, which are less suppressed. The $\mathrm{D}_{\mathrm{s}}^{+}$is of particular interest since it contains a charm and anti-strange quark. A higher production yield for $\mathrm{D}_{\mathrm{s}}^{+}$is expected due to enhanced strangeness production. Energy loss models currently describe the observed suppression at high transverse momentum reasonably well [15] whereas the description at low transverse momentum $(\leq 2 \mathrm{GeV} / c)$ is more challenging (cf. Figure 3, right panel). The data are not well described by next-to-leading-order pQCD calculations with nuclear shadowing (initial state effect) [16], which leads to the conclusion that the observed suppression is indeed a final state effect, arising from the QGP matter. The D meson yields are suppressed at the same level as observed for light-quark hadrons, which was not expected due to the dead-cone and colour-charge effects (cf. Figure 4, left panel). Measurements from the CMS collaboration [17] based on displaced $\mathrm{J} / \psi$ production and beauty-tag jets provide first indications that beauty has indeed smaller energy loss in the QGP than D mesons (cf. Figure 4, right panel).
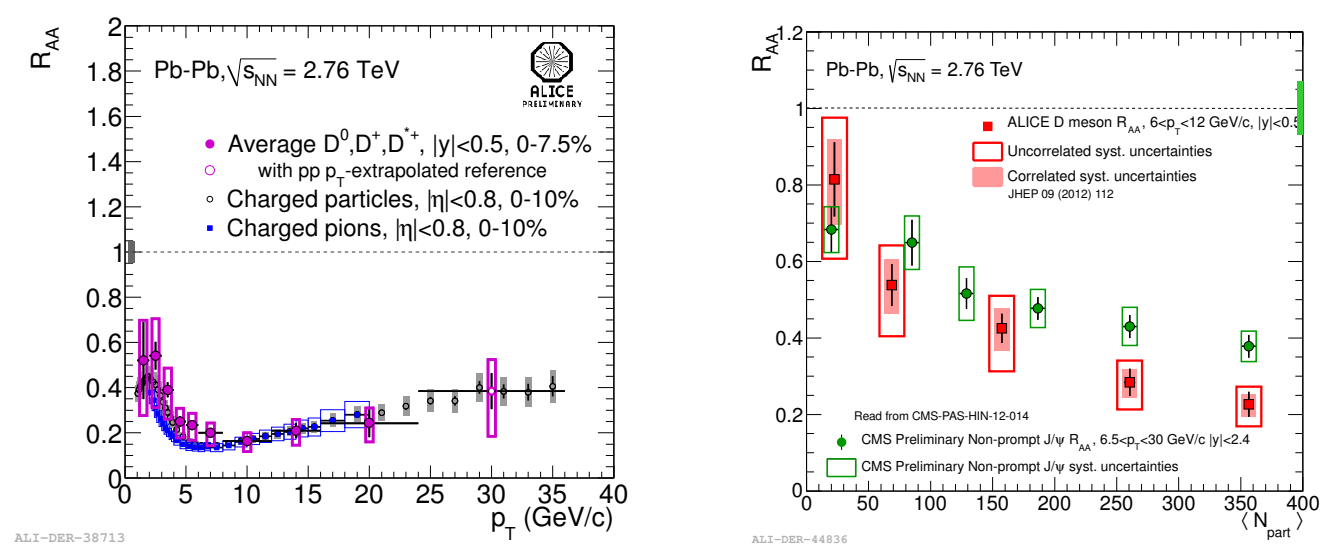

Figure 4: Left: Transverse momentum dependence of the average $R_{\mathrm{AA}}$ of prompt $\mathrm{D}$ mesons at mid-rapidity in the $7.5 \%$ most central lead-lead collisions at $\sqrt{s_{\mathrm{NN}}}=2.76 \mathrm{TeV}$, compared to charged hadrons and pions. Right: $R_{\mathrm{AA}}$ of prompt $\mathrm{D}$ mesons and non-prompt $\mathrm{J} / \psi$ versus the number of participants. 


\section{Summary}

Heavy quarks (charm and beauty) are particularly good probes to study the dynamical properties of the Quark Gluon Plasma, created in high energy heavy-ion collisions. LHC measurements in central lead-lead collisions at $\sqrt{s_{\mathrm{NN}}}=2.76 \mathrm{TeV}$ have shown that the yield of prompt $\mathrm{D}$ mesons, with respect to pp interactions, are suppressed to the same level as observed for light-quark hadrons, leading to the conclusion that these quark flavours have similar interactions with the medium constituents. First indications have be found that beauty experiences less energy loss in the hot QCD medium. More data are needed for the quantitative understanding of fundamental quantities of the QGP such as the transport coefficient.

\section{Acknowledgments}

I thank the ALICE, ATLAS and CMS Collaborations for providing the data and the LHC accelerator team. The European Research Council has provided financial support under the European Community's Seventh Framework Programme (FP7/2007-2013) / ERC grant agreement no 210223. This work was also supported by a Vidi grant from the Netherlands Organisation for Scientific Research (project number 680-47-232) and Projectruimte grants from the Dutch Foundation for Fundamental Research (project numbers: 10PR2884 and 12PR3083).

\section{References}

[1] B. Erazmus for the ALICE Collaboration, these proceedings.

[2] B. Müller, J. Schukraft and B. Wyslouch, Ann. Rev. Nucl. Part. Sci. 62, 361 (2012).

[3] Z. Lin and M. Gyulassy, Phys. Rev. C 51, 2177 (1995).

[4] J. Uphoff, O. Fochler, Z. Xu and C. Greiner, Phys. Rev. C 82, 044906 (2010).

[5] Y. Dokshitzer and D. Kharzeev, Phys. Lett. B 519, 199 (2001).

[6] M. Djordjevic, M. Gyulassy and S. Wicks, Phys. Rev. Lett. 94, 112301 (2005).

[7] The CERN Large Hadron Collider: Accelerator and Experiments, Vol. 1 and 2, Edited by A. Breskin and R. Voss, JINST 3, S08001-S08007 (2008).

[8] B. Abelev et al. (ALICE Collaboration), JHEP 07, 191 (2012).

[9] B. Abelev et al. (ALICE Collaboration), JHEP 01, 128 (2012).

[10] D. Stocco for the ALICE Collaboration, these proceedings.

[11] M. Mangano, P. Nason and G. Ridolfi, Nucl. Phys. B 373, 295 (1992).

[12] T. Sjöstrand and M. van Zijl, Phys. Lett., B 188, 149 (1987).

[13] G. Aad et al. (ATLAS Collaboration), Phys. Rev. D 85, 052005 (2012).

[14] S. Wicks, W. Horowitz, M. Djordjevic and M. Gyulassy, Nucl. Phys. A 784, 426 (2007).

[15] B. Abelev et al. (ALICE Collaboration), JHEP 09, 112 (2012).

[16] M.L. Mangano, P. Nason and G. Ridolfi, Nucl. Phys. B 373, 295 (1992).

[17] C. Mironov for the CMS Collaboration, Nucl. Phys. A 904-905, 194c (2013). 